In summary, we show for the first time that the absence of a trident peak at $7 \mathrm{ppm}$ in NMR spectra of $\mathrm{EBC}$, related to ammonium, is associated with asthma. The loss of ammonium reflects reduced ammonia synthesis due to downregulation of glutaminase, leading to impaired acid neutralisation. Surprisingly, despite a very strong association in our study, this signature was not noted in the seminal study by CARRARO et al. [2], who first described the use of NMR spectroscopy of EBC in asthma. Only spectra between 1 and 4 ppm were shown in their study. One possibility for the observed differences is that CARRARO et al. [2] used a reusable collection system. It is possible, as suggested by IZQUIERDO-GARCíA et al. [4], that the use of disinfectants in reusable collection systems may create artefacts, cleaning of which may in turn obscure some parts of the spectra. As we use a completely disposable tube for EBC collection, with only a chilled external metal sleeve being reused, there is no possibility of such contamination in our data. It was additionally confirmed that EBC spectra including the 7 ppm peaks were distinct from deuterated water condensate collected from identical tubes. Furthermore, independent previous reports of ammonia and ammonium being reduced in asthma corroborate our findings $[5,9]$. The small number of controls in our study, particularly of children, limits our study and further studies are needed to establish the usefulness of our finding. Also, it appears likely that the ammonium signature is enhanced by acidic load on the airway, which may vary across geographical regions. This study was conducted in Delhi, India, where ambient air contains high sulphuric and nitric oxides. Applicability of our finding to less polluted regions needs to be tested. However, despite this, our data hopefully removes the need for any further debate regarding whether valid and useful NMR spectra can be obtained for EBC [4]. This nascent field holds much potential for translation, and concerted efforts of many investigators are needed.

\section{A. Sinha*, V. Krishnan*, T. Sethi*, S. Roy", B. Ghosh*, R. Lodha", S. Kabra" and A. Agrawal*}

${ }^{*}$ Center of Excellence, Translational Research in Asthma and Lung Disease, Council of Scientific and Industrial Research (CSIR) Institute of Genomics and Integrative Biology, "Dept of Paediatrics, All India Institute of Medical Sciences, Delhi, and "Structural Biology and Bioinformatics, CSIR-Indian Institute of Chemical Biology, Kolkata, India.
Correspondence: A. Agrawal, Institute of Genomics and Integrative Biology, Mall Road, Delhi 110007, India. E-mail: a.agrawal@igib.in

Support Statement: The study was funded by the Centre for Excellence Project MLP 5501 of Council of Scientific and Industrial Research (CSIR), Government of India. A. Agrawal acknowledges the support of the Lady Tata Memorial Trust.

Statement of Interest: None declared.

Acknowledgements: The authors acknowledge research funding support from CSIR project MLP5501. They thank members of the Dept of Paediatrics, All India Institute of Medical Sciences, Delhi, especially M.B. Meenakshi, A.P. Anibha, B.J. Bipin and H.P. Hrishikesh, for collection of patient samples and to the Dept of Structural Biology and Bioinformatics at IICB, Kolkata, India, with a special thanks to E.P. Padmanabhan for help in performing NMR spectroscopy.

\section{REFERENCES}

1 Horváth I, Hunt J, Barnes PJ, et al. ATS/ERS Task Force on Exhaled Breath Condensate. Eur Respir J 2005; 26: 523-548.

2 Carraro S, Rezzi S, Reniero F, et al. Metabolomics applied to exhaled breath condensate in childhood asthma. Am J Respir Crit Care Med 2007; 175: 986-990.

3 De Laurentiis G, Paris D, Melck D. Metabonomic analysis of exhaled breath condensate in adults by nuclear magnetic resonance spectroscopy. Eur Respir J 2008; 32: 1175-1183.

4 Izquierdo-García JL, Peces-Barba G, Heili S, et al. Is NMR-based metabolomic analysis of exhaled breath condensate accurate? Eur Respir J 2011; 37: 468-470.

5 Hunt JF, Erwin E, Palmer L, et al. Expression and activity of pHregulatory glutaminase in human airway epithelium. Am J Respir Crit Care Med 2002; 165: 101-107.

6 Reddel HK, Taylor DR, Bateman ED, et al. An Official American Thoracic Society/European Respiratory Society Statement: Asthma Control and Exacerbations. Am J Respir Crit Care Med 2009; 180: 59-99.

7 Alix $\mathrm{O}$, Brown $\mathrm{P}, \mathrm{Ngam}$ trakulpanit L, et al. Normative data for $\mathrm{pH}$ of exhaled breath condensate. Chest 2006; 129: 426-430.

8 Petrun NM, Migal LA, Drannik GN. Activity of glutamine deaminating enzymes in the kidney, liver and serum of dogs with renal failure and under normal conditions. Vopr Med Khim 1977; 23: 485-489.

9 MacGregor G, Ellis S, Andrews J, et al. Breath condensate ammonia is lower in children with chronic asthma. Eur Respir J 2005; 26: 271-276.

\title{
Widespread use of serological tests for tuberculosis: data from 22 high-burden countries
}

\section{To the Editors:}

There is great excitement over the introduction of new tuberculosis (TB) diagnostics [1]. Since 2007, several TB diagnostics and approaches have been endorsed by the World Health Organization (WHO) [2],with Xpert MTB/RIF (Cepheid, Sunnyvale, CA, USA) being the most recent [3]. Amidst this excitement, there is growing concern surrounding the use of inappropriate and suboptimal TB diagnostics [4,5].

Currently available commercial serological (antibody detection) tests for TB are inaccurate and highly inconsistent [6-8]. The International Standards for TB Care explicitly discourage their use [9]. Even so, serological tests are known to be widely used in 


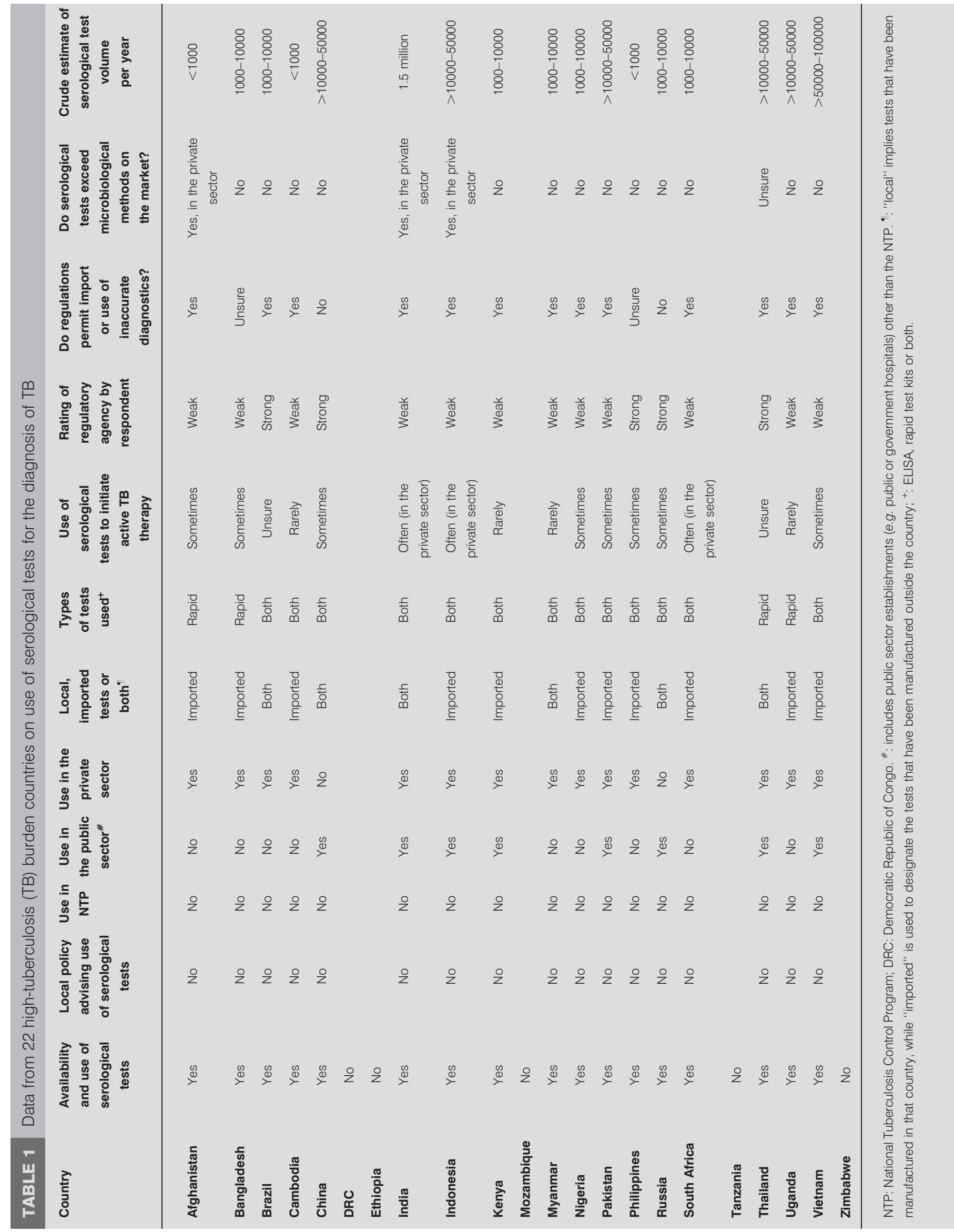


countries such as India $[2,4,5]$. In addition to posing an economic burden on patients and healthcare systems, use of serological tests also entails potential harm to patients (e.g. unnecessary TB therapy because of false-positive results, or morbidity and mortality because of false-negative serology results).

After reviewing the evidence, including the findings of an updated meta-analysis [10], the WHO recently announced its first negative policy in $\mathrm{TB}$, against the use of current $\mathrm{TB}$ serological tests $[5,11]$. In the context of this new policy, we conducted a survey of 22 high-TB burden countries, in order to quantify the current market for serological tests and settings where they are used, and elicit perceptions about regulatory agencies.

We identified TB experts in each country and invited them to complete an electronic e-mailed survey. Although no specific selection criteria were used, we contacted a mix of public and private providers, including experts from national TB programmes. Completed surveys were obtained from at least two respondents in each of the 22 countries. When discrepant responses were noted, surveys were sent to additional experts. Thus, three or four experts were surveyed for some countries. As shown in table 1, respondents from 17 (77\%) out of 22 countries reported that serological tests were on the market and being used.

In 13 out of the 17 countries where serological tests were used, both rapid test kits and ELISA-based assays were available, and all 17 countries had imported tests on the market. The most commonly available tests included MycoDot (Mossman Associates, Millford, MA, USA), ICT TB (ICT Diagnostics, Sydney, NSW, Australia), SD Rapid TB (SD Bio Standard Diagnostics, Gurgaon, India), Pathozyme Myco (Omega Diagnostics, Clackmannanshire, UK), TB IgG, IgM, IgA (J. Mitra \& Co., Delhi, India), and Anda TB (Anda Biologicals, Strasbourg, France).

As shown in table 1, none of the 17 countries had any policies that recommended serological tests. Of these countries, $15(88 \%)$ reported use in the private sector and eight $(47 \%)$ in the public sector (all outside of the National Tuberculosis Control Programme). When asked to rank the most commonly used tests for active $\mathrm{TB}$, respondents in all countries ranked microbiological techniques (sputum smears and cultures) as more frequently used than serological tests, but serological tests were generally ranked higher in the private sector than in the public sector.

Respondents from 11 (65\%) out of 17 countries reported that serological tests were used as the basis for initiating therapy for active TB; those in India, Indonesia and South Africa reported that this happened "often" in the private sector. Respondents from $12(71 \%)$ out of 17 countries perceived their regulatory agencies as being "weak", and 11 acknowledged that such weakness may allow for the importation and/or use of serological tests. Interestingly, some respondents rated their regulatory agencies as "strong", despite the fact that serological tests were available in these countries.

While most respondents were unable to provide much data on the volume or cost, the median cost to a patient for one test was estimated at US \$12 (range \$5-130). India reported the highest volume of testing, an estimated 1.5 million TB serological tests were performed, at an expenditure conservatively estimated at US \$15 million per year. But serological testing was widespread in other countries as well, especially China, Indonesia, Pakistan, Vietnam and Uganda. Further work is necessary to collect more reliable estimates of test volumes and costs.

While the abuse of serological tests in India has received press coverage $[4,5,12-15]$, the problem extends beyond India to most other high-burden countries. Regulation of TB diagnostics is weak in these countries, allowing for poorly performing tests to enter the market. Once on the market, financial gains by stakeholders (doctors, laboratories and diagnostic companies) keep such products profitable [4, 5, 14]. Our survey also confirms the previous observation $[5,14,15]$ that companies in western countries (e.g. France, UK, USA, Germany and Australia) are exporting inaccurate and unreliable $\mathrm{TB}$ diagnostics to poor countries, while not approving the same tests for domestic use.

Misdiagnosis of TB is harmful both to individual patients and to public health; every missed TB diagnosis may result in additional TB transmission. Furthermore, when inaccurate and inappropriate tests are widely used, scale-up of validated diagnostics may be more difficult. While the recent WHO policy against serological TB testing provides important guidance at the global level, it will have no impact unless high-burden countries implement this policy, tighten regulations, and educate doctors, laboratories and consumers to prevent continued abuse of such diagnostics. Countries must also find ways to incentivise the private sector to substitute serological tests with validated WHO-endorsed products, such that the economic incentives of the private sector are more closely aligned with the health incentives of individual TB patients. Lastly, intensive biomarker research is urgently needed to develop accurate and reliable point-of-care serological tests that can replace the existing ineffective assays.

\section{J. Grenier, ${ }^{*}$, L. Pinto ${ }^{*, \#, ~ D . ~ N a i r ~}{ }^{\Uparrow}$, K. Steingart ${ }^{+}$, D. Dowdy ${ }^{\S}$, A. Ramsay ${ }^{\dagger}$ and M. Pai ${ }^{*, \#}$}

*Respiratory Epidemiology and Clinical Research Unit, Montreal Chest Institute, "Dept of Epidemiology and Biostatistics, McGill University, Montreal, QC, Canada, "Dept of Microbiology, Vardhman Mahavir Medical College and Safdarjung Hospital, New Delhi, India, ${ }^{+}$University of Washington School of Public Health, Seattle, WA, ${ }^{\S}$ Dept of Epidemiology, Johns Hopkins University School of Public Health, Baltimore, MD, USA, and ${ }^{f}$ UNICEF/UNDP/World Bank/WHO Special Programme for Research and Training in Tropical Diseases, World Health Organization, Geneva, Switzerland.

Correspondence: M. Pai, McGill University, Dept of Epidemiology and Biostatistics, 1020 Pine Ave West, Montreal, QC H3A 1A2, Canada. E-mail: madhukar.pai@mcgill.ca

Support Statement: This study was supported, in part, by a grant from the Canadian Institutes of Health Research (CIHR MOP89918) and the European and Developing Countries Clinical Trials Partnership (TB-NEAT grant). These agencies had no role in the design, execution or publication of this study. J. Grenier was supported by a Meakins-Christie Summer Studentship, L. Pinto was supported by a McGill University Health Center Research Institute fellowship award and M. Pai was supported by a New Investigator Award from CIHR. M. Pai is a consultant 
to the Bill \& Melinda Gates Foundation (BMGF). BMGF had no involvement in this publication.

Statement of Interest: Statements of interest for K. Steingart, A. Ramsay and M. Pai can be found at www.erj.ersjournals.com/ site/misc/statements.xhtml

Acknowledgements: We are very grateful to the more than $50 \mathrm{~TB}$ experts who completed our surveys or provided input. We remain responsible for any errors.

\section{REFERENCES}

1 Small PM, Pai M. Tuberculosis diagnosis - time for a game change. N Engl J Med 2010; 363: 1070-1071.

2 Pai M. Improving TB diagnosis: difference between knowing the path and walking the path. Expert Rev Mol Diagn 2011; 11: 241-244.

3 World Health Organization. Roadmap for Rolling Out Xpert MTB/RIF for Rapid Diagnosis of TB and MDR-TB. Geneva, World Health Organization, 2010.

4 Specter M. A Deadly Misdiagnosis. Why Millions of People Still Die from TB? New Yorker. November 15, 2010.

5 Morris K. WHO recommends against inaccurate tuberculosis tests. Lancet 2011; 377: 113-114.

6 Steingart KR, Henry M, Laal S, et al. A systematic review of commercial serological antibody detection tests for the diagnosis of extra-pulmonary tuberculosis. Thorax 2007; 62: 911-918.

7 Steingart KR, Henry M, Laal S, et al. Commercial serological antibody detection tests for the diagnosis of pulmonary tuberculosis: a systematic review. PLoS Med 2007; 4: e202.
8 World Health Organization. Diagnostics Evaluation Series No.2. Laboratory-Based Evaluation of 19 Commercially Available Rapid Diagnostic Tests for Tuberculosis. Geneva, World Health Organization, 2008.

9 Hopewell PC, Pai M, Maher D, et al. International standards for tuberculosis care. Lancet Infect Dis 2006; 6: 710-725.

10 Steingart KR, Flores L, Dendukuri N, et al. Commercial serological tests for the diagnosis of active pulmonary and extrapulmonary tuberculosis: an updated systematic review and meta-analysis. PLoS Med 2011; 8: e1001062.

11 World Health Organization. Commercial serodiagnostic tests for diagnosis of tuberculosis: policy statement. http:/ / whqlibdoc.who. int/publications/2011/9789241502054_eng.pdf Date last accessed: November 4, 2011. Date last updated: 21 July 2011.

12 Mudur GS. India alert against TB blood tests. www.telegraphindia. com/1110721/jsp/nation/story_14268228.jsp Date last accessed: November 4, 2011. Date last updated: July 21, 2011.

13 Mascarehnas A. Scientists warn against blood tests for TB, say inaccurate. www.expressindia.com/latest-news/Scientists-warn-againstblood-tests-for-TB-say-inaccurate/837274/ Date last accessed: November 4, 2011. Date last updated: August 26, 2011.

14 Pai M, Dowdy D, Steingart KR. Medical, public health and economic consequences of bad diagnostics for tuberculosis. http://blogs.plos. org/speakingofmedicine/2011/08/09/medical-public-health-andeconomic-consequences-of-bad-diagnostics-for-tuberculosis/ Date last accessed: November 4, 2011. Date last updated: August 9, 2011.

15 Chinnock P. The Scandal of TB Misdiagnosis. www.tropika.net/ svc/news/20101113/Chinnock-20101113-News-TB-India Date last accessed: November 4, 2011. Date last updated: November 13, 2010.

DOI: 10.1183/09031936.00070611

\section{Palliative percutaneous cryoablation in a patient with locally advanced invasive thymoma}

\section{To the Editors:}

Percutaneous cryoablation is considered to be a minimally invasive option for local treatment of cancer [1-3]. Its efficacy is now being documented in many organs, including the prostate, liver, kidney and lung. The goal of ablation procedures is typically complete local tumour control when surgical resection is not indicated, but ablation procedures, such as cryoablation, may also be applicable to the palliative care of cancer patients. Here, we present a case in which percutaneous cryoablation was considered to have contributed to symptom relief in a patient with unresectable, locally advanced, invasive thymoma of the mediastinum. To our knowledge, this is the first report of a needle ablation procedure performed for a mediastinal tumour.

A 29-yr-old female presented with intermittent cough that had existed for 2 yrs and had gradually worsened. An abnormal shadow on chest radiography was identified at another clinic and the patient was referred to our hospital (Division of General Thoracic Surgery, Dept of Surgery, School of Medicine, Keio University, Tokyo, Japan) in July 2004. At that time, she complained of frequent cough and dyspnoea. She could not lay flat on her back. Biopsy through parasternal mediastinotomy $[4,5]$ was performed, from which the pathological diagnosis was thymoma, type B3, according to the World Health Organization classification. The tumour was considered to be unresectable because it had encased the major vessels and the airway in the mediastinum (fig. 1a-c). Two courses of carboplatin plus VP-16 with concurrent radiation (total $50 \mathrm{~Gy}$ ) [6] were administered. Radiologically, no apparent changes were seen, but her cough and dyspnoea were worse 1 month after chemoradiotherapy. Facial oedema had also appeared. We speculated that progressive compression of the trachea, carina and superior vena cava by the tumour was, at least in part, responsible for these symptoms. Since chemoradiotherapy appeared to be ineffective at this point, we decided to attempt tumour volume reduction by cryoablation, primarily to reduce airway compression. In November 2004, percutaneous cryoablation was performed. The cryoablation protocol had been approved by the institutional review board in October 2002. Written consent was obtained from the patient.

The percutaneous cryoablation procedure was performed under local anaesthesia as previously described [1]. Under a multidetector row computed tomography $(\mathrm{CT})$ scanner with multislice CT fluoroscopy functions (Aquilion 64; Toshiba Med. Co. Ltd, Tokyo, Japan), using an outer insertion sheath, a 1.7-mm diameter cryoprobe (CRYOcare Cryosurgical Unit; Endocare, Irvine, CA, 